\title{
SPECIAL SHORT WAVE FINITE ELEMENTS FOR FLOW ACOUSTICS
}

\author{
Jeremy Astley, Pablo Gamallo \\ Institute of Sound and Vibration Research, Univ. of Southampton, Highfield, SO17 1BJ, Southampton, UK
}

Summary A numerical scheme based on the Partition of Unity Finite Element Method is proposed for solving shortwave problems in flow acoustics. The goal of this approach is to obtain accurate solutions with fewer degrees of freedom than are required for conventional grid or node based schemes. Preliminary numerical results obtained for 2-D and axisymmetric models indicate that this approach may offer significant performance improvements.

\section{INTRODUCTION}

Situations in which wavelike disturbances propagate with wavelengths which are small compare to the dimensions of the solution domain are common in many branches of engineering and physics. The work reported here focusses on a particular problem of this type, that of sound propagation in the ducted regions of turbofan aircraft engines, where the diameter of the engine is generally an order of magnitude larger than the characteristic wavelength of the sound field. The (subsonic) Mach number within such ducts also varies giving rise to significant inhomogeneous propagation effects. Although the current analysis is directed specifically at this problem, the general approach that is proposed can be applied to a wider class of problems which involve convective wave propagation (surface gravity waves on nonuniform currents for example).

When traditional Finite Element (FE) models are applied to short wave problems, approximability considerations alone dictate a minimum resolution of 8 - 10 nodes per wavelength, a requirement which is exacerbated by 'pollution' error [2] when many wavelength variations are present in the computational domain. Moreover, when flow is present, wave-shortening due to the mean flow may further increase mesh requirements.

Aero-engine short wave FE computations can currently be performed at acceptable computational cost only by using axisymmetric models $[3,1]$. These generally contain of the order of $10^{5}$ degrees of freedom. The same approach becomes prohibitively expensive for fully 3-D problems, even when parallel codes are used [6].

In this article a novel method is proposed for the solution of such problems. It is based on the Partition of Unity Finite Element Method (PUFEM) [5] and uses very sparse 'meshes' ( $<1$ node per wavelength). The method is an extension to flow acoustics of the PUFEM approach which has been applied to the standard Helmholtz equation [4] and shown to be relatively free of pollution error. The method is based on a discrete set of local plane wave solutions which are used to 'enrich' a conventional finite element trial space. The method is implemented in the frequency domain using a weak variational formulation.

\section{FORMULATION OF THE PROBLEM}

We consider an inviscid, isentropic, compressible fluid in which a small time-harmonic acoustic disturbance propagates upon a steady irrotational mean flow. The acoustic field is represented by an acoustic velocity potential $\phi(\mathbf{x}) e^{i \omega t}$ consistent with an assumption of an irrotational mean flow. The linearised mass and momentum equations yield the following convected acoustic wave equation

$$
\omega^{2} \frac{\rho_{0}}{c_{0}^{2}} \phi-i \omega\left(\frac{\rho_{0} \mathbf{u}_{\mathbf{0}}}{c_{0}^{2}} \cdot \nabla \phi+\nabla \cdot\left(\frac{\rho_{0} \mathbf{u}_{\mathbf{0}}}{c_{0}^{2}} \phi\right)\right)-\nabla \cdot\left(\left(\frac{\rho_{0} \mathbf{u}_{\mathbf{0}}}{c_{0}^{2}} \cdot \nabla \phi\right) \mathbf{u}_{\mathbf{0}}\right)+\nabla \cdot\left(\rho_{0} \nabla \phi\right)=0,
$$

where $\rho_{0}, p_{0}, c_{0}$, and $\mathbf{u}_{0}=\nabla \phi_{0}$ are the density, pressure, sound speed and flow velocity of the mean flow. A weak formulation of the above equation forms the basis for both conventional FE and PUFEM analyses.

\section{THE PARTITION OF UNITY FINITE ELEMENT METHOD (PUFEM)}

The Partition of Unity method - first proposed by Melenk and Babuška [5] - allows information about the local physical solution to be included in an approximation subspace. In the case of equation (1), a finite set of plane wave solutions defined at nodal points is used to build a discrete trial space. By setting the mean flow Mach number to a constant (axial) value $M$, equation (1) becomes

$$
\frac{\partial^{2} \phi}{\partial y^{2}}+\left(1-M^{2}\right) \frac{\partial^{2} \phi}{\partial x^{2}}-2 i k M \frac{\partial \phi}{\partial x}+k^{2} \phi=0
$$

and a family of exact local solutions is defined by

$$
\phi(x, y)=A e^{-i\left(k_{x} x+k_{y} y\right)}=A e^{-i k \frac{(x \cos \theta+y \sin \theta)}{1+M \cos \theta}},
$$

where $k=\omega / c_{0}, A \in \mathbb{C}$ and $\theta \in[0,2 \pi)$ is the angle between the flow velocity and the wave direction. The convective effect of the mean flow gives a different wave speed for each direction and hence a wavenumber which varies with $\theta$. In the case of non-uniform mean flow, the local solutions are orientated in the direction of the 

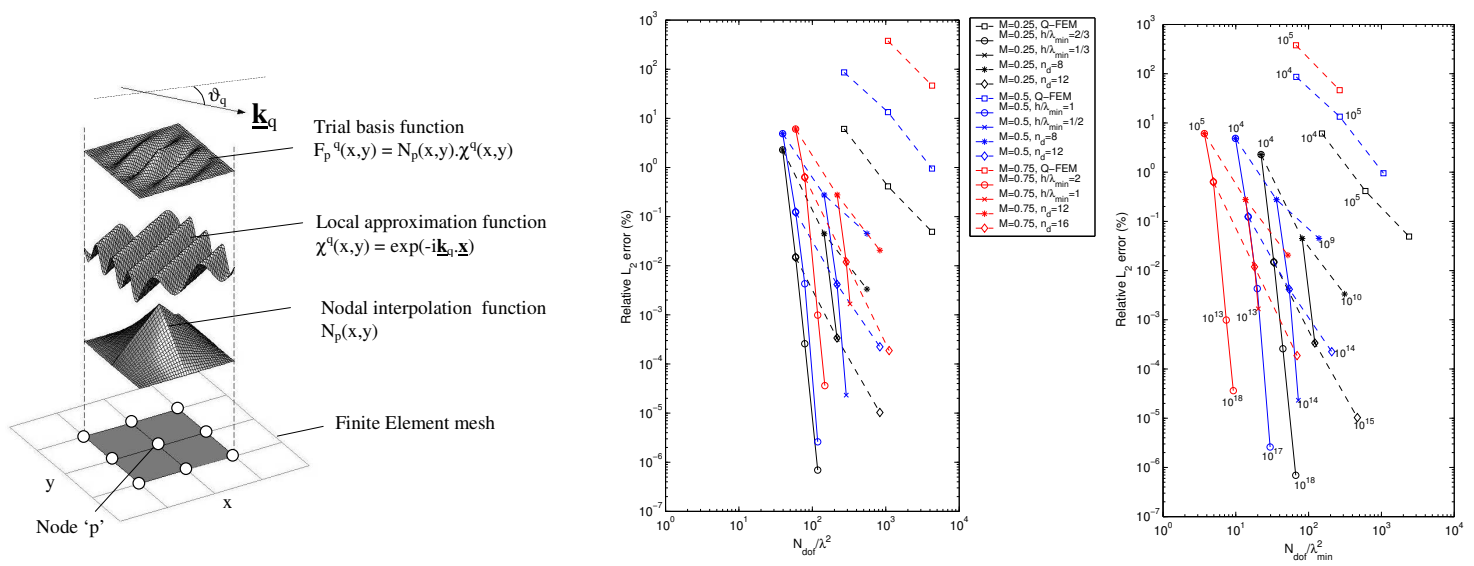

Figure 1: Construction of the PUFEM trial basis (left). Results for a lined 2-D uniform duct (right).

mean flow. The PUFEM discrete trial space is then constructed by selecting a finite number of wave directions, $n_{d}(l)$ say, at each node $l$ of a conventional FE mesh. A generic element $\phi_{h}$ of the PUFEM finite element space is written

$$
\phi_{h}(x, y)=\sum_{l=1}^{N_{\text {nodes }}} N_{l}(x, y) \sum_{j=1}^{n_{d}(l)} A_{l}^{j} e^{-i k \frac{\left(x \cos \theta_{j}+y \sin \theta_{j}\right)}{1+M \cos \theta_{j}}}, \quad \text { with } \sum_{l=1}^{N_{\text {nodes }}} N_{l}(x, y)=1 \text { (partition of unity) }
$$

where $N_{\text {nodes }}$ is the number of nodes in the mesh, $N_{l}(x, y)\left(l=1, \cdots, N_{\text {nodes }}\right)$ are the conventional nodal shape functions and $A_{l}^{j}\left(l=1, \cdots, N_{\text {nodes }}\right.$ and $\left.j=1, \cdots, n d(l)\right)$ are the unknown nodal amplitudes. These form the d.o.f. for the model. i.e. A typical PUFEM trial basis function is the product of a shape function and a wave function as illustrated in the left hand picture in Figure 1. A weak variational formulation is used to obtain a set of discrete algebraic equations for the unknown variables $A_{l}^{j}$.

Results are presented in Figure 1 for a 2-D test case in which prescribed modes propagate in a uniform lined duct. The $L_{2}$ error in the computed solution is plotted against a resolution parameter $N_{d o f} / \lambda^{2}$. Results are shown for a reduced frequency $k a=20$ ( $a$ is the duct width) with $M=0.25,0.5,0.75$. Two definitions of the wavelength $\lambda$ are considered in the parameter $N_{d o f} / \lambda^{2}$; the mean value in the left hand graph, and the minimum (i.e upstream) value in the right hand graph. The condition number of the coefficient matrix is noted beside selected data points. The accuracy of the solution can be improved either by refining the mesh $(h$ refinement, indicated by the dashed lines) or by increasing the number of wave directions at each node (a sort of 'p' refinement, indicated by solid lines). Clearly the improvement due to 'p' refinement is more rapid but also increases the condition number. Also shown are data for a series of conventional quadratic FE solutions (Q-FEM). All of the PUFEM models offer a significant improvement in accuracy over the conventional FE model for a given problem size. Results (not shown) indicate that the same conclusions hold for nonuniform ducts.

\section{CONCLUSIONS}

The PUFEM approach has given promising results for 2-D test problems. These incorporate many aspects of propagation in 'real' inlet and bypass ducts (non-uniform geometry, flow gradients and liners) but further studies are needed to determine whether PUFEM can deliver the execution times which are needed for practical three dimensional problems.

\section{References}

[1] R. J. Astley, J. A. Hamilton, N. Baker, and E. Kitchen. Modelling tone propagation from turbofan inlets the effect of extended lip liners. AIAA paper 2002-2449, 2002.

[2] A. Deraemaeker, I. Babuska, and P. Bouillard. Dispersion and pollution of the FEM solution for the Helmholtz equation in one, two and three dimensions. 46:471-499, 1999.

[3] W. Eversman. Mapped infinite wave envelope elements for acoustic radiation in a uniformly moving medium. J. Sound Vib., 224:665-687, 1999.

[4] O. Laghrouche, P. Bettess, and R. J. Astley. Modelling of short wave diffraction problems using approximating systems of plane waves. Int. J. Numer. Meth. Engng., 54:1501-1533, 2002.

[5] J.M. Melenk and I. Babuška. The partition of unity finite element method: Basic theory and applications. Comput. Methods Appl. Mech. Engrg., 139:289-314, 1996.

[6] D. Stanescu, M. Y. Hussaini, and F. Farassat. Aircraft engine noise scattering - a discontinuous spectral element approach. AIAA paper 2002-0800, 2002. 